\title{
Evidence for an oncogenic role of HOXC6 in human non-small cell lung cancer
}

\author{
Yingcheng Yang Equal first author, 1 ， Xiaoping Tang Equal first author, ${ }^{1}$, Xueqin Song ${ }^{1}$, Li Tang ${ }^{1}$, Yong Cao ${ }^{1}$, Xu Liu ${ }^{1}$, \\ Xiaoyan Wang ${ }^{1}$, Yan Li ${ }^{1}$, Minglan Yu ${ }^{1}$, Feng Chen ${ }^{\text {Corresp., }}{ }^{1}$, Haisu Wan ${ }^{\text {Corresp. } 1}$ \\ ${ }^{1}$ Experimental Medicine Center, The Affiliated Hospital of Southwest Medical University, Luzhou, Sichuan, China \\ Corresponding Authors: Feng Chen, Haisu Wan \\ Email address: chenf6305@foxmail.com,whssyzx@swmu.edu.cn
}

Background: Identification of specific biomarkers is important for the diagnosis and treatment of nonsmall cell lung cancer (NSCLC). HOXC6 is a homeodomain-containing transcription factor that is highly expressed in several human cancers; however, its role in NSCLC remains unknown.

Methods: The expression and protein levels of HOXC6 were assessed in NSCLC tissue samples by Quantitative real-time PCR (qRT-PCR) and immunohistochemistry, respectively. HOXC6 was transfected into the NSCLC cell lines A549 and PC9, and used to investigate its effect on proliferation, migration, and invasion using CFSE, wound healing, and Matrigel invasion assays. Next-generation sequencing was also used to identify downstream targets of HOXC6 and to gain insights into the molecular mechanisms underlying its biological function.

Results: HOXC6 expression was significantly increased in $66.6 \%(20 / 30)$ of NSCLC tumor samples in comparison to normal controls. HOXC6 promoted proliferation, migration, and invasion of NSCLC cells in vitro. RNA-seq analysis demonstrated the upregulation of 310 and 112 genes in A549-HOXC6 and PC9HOXC6 cells, respectively, and the downregulation of 665 and 385 genes in A549-HOXC6 and PC9-HOXC6 cells, respectively. HOXC6 was also found to regulate the expression of genes such as CEACAM6, SPARC, WNT6, CST1, MMP2, and KRT13, which have documented pro-tumorigenic functions.

Discussion: HOXC6 is highly expressed in NSCLC, and it may enhance lung cancer progression by regulating the expression of pro-tumorigenic genes involved in proliferation, migration, and invasion. Our study highlighted the oncogenic potential of HOXC6, and suggests that it may be a novel biomarker for the diagnosis and treatment of NSCLC. 


\section{Evidence for an oncogenic role of HOXC6 in human non- 2 small cell lung cancer}

3

4 Yingcheng Yang ${ }^{1}$, Xiaoping Tang ${ }^{1}$, Xueqin Song ${ }^{1}$, Li Tang ${ }^{1}$, Yong $\mathrm{Cao}^{1}$, Xu Liu ${ }^{1}$, Xiaoyan

5 Wang $^{1}$, Yan $\mathrm{Li}^{1}$, Minglan $\mathrm{Yu}^{1}$, Feng Chen ${ }^{1}$ and Haisu Wan ${ }^{1}$

6

$7{ }^{1}$ Experimental Medicine Center, The Affiliated Hospital of Southwest Medical University, 8 Luzhou, Sichuan, China.

10 Corresponding Authors:

11 Haisu Wan ${ }^{1}$

12 No. 25 Taiping Street, Luzhou, Sichuan, 646000, China

13 Email address: whssyzx@swmu.edu.cn

14 Feng Chen ${ }^{1}$

15 No. 25 Taiping Street, Luzhou, Sichuan, 646000, China

16 Email address: chenf6305@,foxmail.com 


\section{Abstract}

Background: Identification of specific biomarkers is important for the diagnosis and treatment of non-small cell lung cancer (NSCLC). HOXC6 is a homeodomain-containing transcription factor that is highly expressed in several human cancers. However, its role in NSCLC remains unknown.

Methods: The gene expression and protein levels of HOXC6 were assessed in NSCLC tissue samples using quantitative real-time PCR (qRT-PCR) and immunohistochemistry, respectively. HOXC6 was transfected into the NSCLC cell lines A549 and PC9, and then used to investigate its effect on proliferation, migration, and invasion using CFSE, wound healing, and Matrigel invasion assays, respectively. Next-generation sequencing was also used to identify downstream targets of HOXC6 and to gain insights into the molecular mechanisms underlying its biological 46 function.

47 Results: HOXC6 expression was significantly increased in 66.6\% (20/30) of NSCLC tumor 48 samples in comparison to normal controls. HOXC6 promoted proliferation, migration, and 49 invasion of NSCLC cells in vitro. RNA-seq analysis demonstrated the upregulation of 310 and 112 genes in A549-HOXC6 and PC9-HOXC6 cells, respectively, and the downregulation of 665 and 385 genes in A549-HOXC6 and PC9-HOXC6 cells, respectively. HOXC6 was also found to regulate the expression of genes such as CEACAM6, SPARC, WNT6, CST1, MMP2, and KRT13, 53 which have documented pro-tumorigenic functions. 
54 Discussion: HOXC6 is highly expressed in NSCLC, and may enhance lung cancer progression 55 by regulating the expression of pro-tumorigenic genes involved in proliferation, migration, and 56 invasion. Our study highlighted the oncogenic potential of $H O X C 6$, and suggests that it may be a 57 novel biomarker for the diagnosis and treatment of NSCLC.

\section{Keywords}

HOXC6, non-small cell lung cancer, molecular marker, bioinformatics, malignancy

60

61

62

63

64

65

66

67

68

69

70

71

72

73

74

75

76

77

78

79

80

81

82

83

84

85

Lung cancer has the highest cancer incidence in the world (Chen et al. 2016b). Non-small cell lung cancer (NSCLC) accounts for $80-85 \%$ of the total number of lung cancer cases (Lee et al. 2013; Zienolddiny \& Skaug 2011). In spite of improvements in imaging science, radical surgical resection, and NSCLC auxiliary detection technologies, most patients have advanced disease at diagnosis, and the overall 5-year survival rate is less than 15\% (National Lung Screening Trial Research et al. 2011; Ye \& Zhao 2016). Therefore, more effective molecular makers are needed for the diagnosis and treatment of NSCLC.

$H O X$ genes belong to the homeobox gene superfamily (Cillo et al. 2001). The human $H O X$ gene family is made up of 39 members in 4 clusters (A-D) located on chromosomes 7, 17, 12, and 2, with each cluster containing 9 to 13 loci (Apiou et al. 1996). Many $H O X$ genes have been found to be closely associated with the progression of cancer (Shah \& Sukumar 2010). HOXA10 can inhibit the invasion of breast cancer cells by enhancing the expression of TP53 (Chu et al. 2004). $H O X B 7$ can also promote tumor growth by upregulating the expression of angiogenic growth factors (Care et al. 2001). HOXC6 is a transcription factor that regulates cell differentiation during embryonic development (Maroulakou \& Spyropoulos 2003). Aberrant expression of HOXC6 may result in the malignant transformation of normal cells (DeInnocentes et al. 2015;

Feng et al. 2009; Moon et al. 2012; Wright et al. 1989; Zhang et al. 2013), and elevated HOXC6 expression has been observed in several types of cancers, including prostate, gastrointestinal, colorectal, and hepatocellular cancers (Chen et al. 2016a; Ji et al. 2016; Sui et al. 2016;

Vinarskaja et al. 2011). However, the biological function of HOXC6 has not been well understood. Here, we report that HOXC6 is highly expressed in NSCLC cells, and overexpression of HOXC6 promotes the proliferation, migration, and invasion of NSCLC cells. The phenotypic effects of HOXC6 may be mediated by genes that have been previously reported to be involved in the progression of cancer. Our data also suggest that HOXC6 is a potential molecular marker for the diagnosis and treatment of NSCLC. 
87 Cell lines

88 NSCLC cell lines A549 and PC9 were obtained from the Stem Cell Bank of the Chinese 89 Academy of Sciences. A549 was maintained in RPMI 1640 Medium (Gibco, Thermo Fisher Scientific, Waltham, MA, USA). PC9 and 293FT cells were maintained in Dulbecco's Modified Eagle's Medium (DMEM) (Gibco, Thermo Fisher Scientific, Waltham, MA, USA), supplemented with 10\% fetal bovine serum (Gibco, Thermo Fisher Scientific, Waltham, MA, USA). All of the cell lines were housed in $37^{\circ} \mathrm{C}$ incubators with $5 \% \mathrm{CO}_{2}$ saturation.

\section{Human clinical specimens}

Clinical specimens were collected from patients at the Affiliated Hospital of Southwest Medical University (Luzhou, China). Tissue samples were surgically retrieved from NSCLC patients after obtaining written consent and with the approval of the ethics committee of the Affiliated Hospital of Southwest Medical University (k2018003-r). Clinical samples were immediately separated into lung tumor tissues $(\mathrm{T})$ and adjacent non-tumor lung tissues $(\mathrm{N})$, flash frozen in liquid nitrogen, and then stored at $-80^{\circ} \mathrm{C}$ until further analysis.

\section{Immunohistochemistry (IHC)}

102

103

104

105

106

107

108

109

110

111

112

113

114

115

116

117

118

119

Formalin-fixed paraffin-embedded sections were treated with $3 \% \mathrm{H}_{2} \mathrm{O}_{2}$ for 10 minutes after deparaffinization in xylene and rehydration in decreasing concentrations of ethanol from 100 to $75 \%$. Antigen retrieval was performed by using heated sodium citrate. Sections were then blocked using $10 \%$ normal goat serum to prevent non-specific antibody reactions. The sections were then incubated overnight at $4^{\circ} \mathrm{C}$, with a mouse monoclonal antibody against human HOXC6 (Santa Cruz, CA, USA, dilution, 1:200). After incubation, the HOXC6 antigen-antibody reaction was performed using an immunoperxodase-based kit (ZSGB Bio, Beijing, China). To quantify the expression of HOXC6, two specialist pathologists scored the stained samples based on the product of the intensity and degree of staining $(0-100 \%)$. The staining intensity was categorized as follows: 0 (negative), 1 (weak), 2 (moderate), and 3 (strong). Only cytoplasmic staining was counted as positive staining.

\section{RNA isolation and Quantitative Real-Time Polymerase Chain Reaction (qRT-PCR)}

Total RNA was extracted from NSCLC cell lines and human samples using TRIzol (TaKaRa Bio, Otsu, Japan). Then, $0.5 \mu \mathrm{g}$ total RNA was reverse transcribed into cDNA using a PrimeScript ${ }^{\mathrm{TM}}$ RT reagent Kit with gDNA Eraser (TaKaRa Bio, Otsu, Japan) according to the manufacturer's instructions. qRT-PCR was performed using SYBR ${ }^{\circledR}$ Premix Ex Taq ${ }^{\mathrm{TM}}$ II (TaKaRa Bio, Otsu, Japan) system on an ABI QuantStudio ${ }^{\text {TM }} 7$ Flex Real-Time PCR (Applied Biosystems, USA). For the PCR reaction, specific primers were selected based on PrimerBank 
120 HOXC6 sequences. The following primers were synthesized by Invitrogen: human HOXC6

121 forward primer 5'-ACAGACCTCAATCGCTCAGGA-3', and reverse primer 5'-

122 AGGGGTAAATCTGGATACTGGC-3'; GAPDH forward primer 5'-

123 ATGCTGGCGCTGAGTACGTC-3', and reverse primer 5'-

124 GGTCATGAGTCCTTCCACGATA-3'. Gene expression levels were normalized to GAPDH 125 using the $2^{-\Delta \Delta C t}$ method.

126 Lentivirus packaging

127 The lentiviral HOXC6-expressing vector pCDH-HOXC6 and empty control vector pCDH-NEO

128 were obtained from Experimental Medicine Center, The Affiliated Hospital of Southwest

129 Medical University. The 293FT cells were used for lentiviral packaging. A total of 3 vectors

130 (pCDH-HOXC6 or pCDH-NEO, psPAX2 and pMD2G) were co-transfected into 293FT cells

131 using Lipofectamine3000 (Invitrogen, Carlsbad, CA, USA). After 72 h, viral supernatant was

132 collected and stored at $-80^{\circ} \mathrm{C}$.

133 Cell transfection

134 A549 and PC9 cells were plated into a 6-well plate. Cells were then transfected with the 135 lentiviral plasmids at $80 \%$ confluence. After $8 \mathrm{~h}$, the transfection medium was replaced with 136 complete growth medium supplemented with $500 \mu \mathrm{g} / \mathrm{ml}$ neomycin. qRT-PCR and Western blot 137 were performed to evaluate transfection efficiency.

\section{Immunoblotting}

139 Cells were lysed on ice using RIPA buffer (Cell Signaling Technology, USA). Protein

140 concentration was measured by BCA kit (Beyotime, Shanghai, China). Equal amounts of total

141 protein were resolved using 12\% SDS-PAGE gel and transferred onto PVDF membranes

142 (Millipore, USA). Membranes were incubated with anti-HOXC6 (Santa Cruz, CA, USA, dilution,

143 1:1000) and anti- $\beta$-actin (Santa Cruz, CA, USA, dilution, 1:1000) overnight at $4^{\circ} \mathrm{C}$ followed by

144 incubation with a secondary antibody. Protein bands were visualized using ECL

145 chemiluminescence (Millipore, Billerica, MA, USA) according to the manufacturer's

146 recommendation.

147 Cell proliferation assay

148 Cell proliferation was performed using Carboxyfluorescein Diacetate Succinimidyl Ester (CFSE)

149 flow cytometry analysis on a BD FACSVerse ${ }^{\mathrm{TM}}$ (BD Biosciences, USA). A final concentration

150 of $5 \mu \mathrm{M}$ CFSE was added to a single cell suspension of $10 \times 10^{6}$ cells $/ \mathrm{ml}$. The dye-cell

151 suspension was then incubated in a $37^{\circ} \mathrm{C}$ water bath for $10-15$ minutes and then analyzed by

152 flow cytometry. Cell proliferation was also assessed using the IncuCyte Live Cell Imaging

153 System. Cells were plated in IncuCyte ImageLock 96-well plates (Essen BioScience, USA) at 1

$154 \times 10^{4}$ cells per well. Cell confluency was monitored using the IncuCyte Live Cell Imaging

155 System (Essen Bioscience, USA) every 2 hours. 
156 Cell migration and invasion assay

157 Transfected cells were plated in IncuCyte ImageLock 96-well plates (Essen BioScience, USA).

158 After cells adhered to the wells, an approximately $600-\mu \mathrm{m}$ wide scratch was made in the cell

159 layer using a WoundMaker (Essen Bioscience, USA). The cells were then washed twice with

160 PBS to remove floating cells. To assess migration, $100 \mu 1$ of growth medium (with $2 \%$ FBS) was

161 added to the cells. For invasion, the plate was coated with $30 \%$ Matrigel for 30 minutes at $37^{\circ} \mathrm{C}$,

162 with an additional $50 \mu 1$ of normal growth medium overlaid on to the plates. Wound closure was

163 monitored using the IncuCyte Live Cell Imaging System (Essen Bioscience, USA) every 2

164 hours. Data analysis was conducted using Incucyte 2016A software.

165 Construction of Sequence libraries

166 Total RNA was extracted from NSCLC cells A549 and PC9. Dynabeads Oligo (dT) 25 beads

167 were used for isolating mRNA. RNA libraries were constructed with NEBNext ${ }^{\circledR}$ Ultra $^{\mathrm{TM}}$

168 Directional RNA Library Prep Kit for Illumina ${ }^{\circledR}$ (NEB, USA) with 3 independent replicates

169 following the manufacturer's instructions. Paired-end sequencing (150bp) was performing using

170 Illumina HiSeq Xten. Raw data was uploaded to the GEO database (GSE121896).

171 Bioinformatics analysis

172 We used HISAT2 (version 2.1.0) (Kim et al. 2015) with default parameters to map the RNA-seq

173 data to the GRCh37.p13 genome from GENCODE (Harrow et al. 2012). We aggregated the read

174 counts at the gene level using HTseq (Anders et al. 2015) and called differentially expressed

175 genes (DEGs) with R package DESeq2 (Love et al. 2014). Genes were considered significantly

176 differentially expressed when the Log2 fold change was $>1$ or $<-1$, and the adjusted $\mathrm{P}$ value was

$177<0.05$. Gene Ontology analysis of the DEGs was performed using the R package ClusterProfiler

178 (Yu et al. 2012) with $\mathrm{P}<0.05$.

179 Statistical analysis

180 All of the statistical analyses were carried out using GraphPad Prism 7 and SPSS 21.0.

181 Numerical data are presented as mean \pm standard error, and were calculated using two-tailed

182 Student's $t$ test and Chi-square test. Two-way analysis of variance (ANOVA) was used to assess

183 the statistical significance of multiple continuous variables. $\mathrm{P}<0.05$ was considered statistically

184 significant.

185 Results

186 High expression of HOXC6 in Human NSCLC tissues

187 We examined the expression level of HOXC6 in lung cancer using in silico approaches. Analysis

188 of publicly available data revealed that there was a 5-fold and 8.8-fold increase in the expression

189 of HOXC6 in 2 independent lung cancer datasets, GSE30219 and GSE27262, respectively (Fig. 
190 1A and 1B). To validate these findings, we used qRT-PCR to determine HOXC6 expression in 191 tumor samples from NSCLC patients. As shown in Figure 1C, in 30 pairs of NSCLC tissue 192 samples and adjacent non-tumor tissue samples, 66.6\% (20/30) had elevated levels of HOXC6 in 193 comparison to their adjacent normal counterparts $(\mathrm{P}=0.01)$. IHC analysis was consistent with the 194 gene expression data, as HOXC6 protein was elevated in NSCLC tumor samples in comparison to adjacent normal controls (Fig. 1D and 1E). These results indicate that HOXC6 is highly expressed in NSCLC tissues.

\section{The effect of HOXC6 overexpression on the proliferation of NSCLC cells}

To further explore the biological function of $H O X C 6$, we used lentiviral vectors to establish HOXC6-overexpressing NSCLC cell lines. The resulting cell lines were named A549-HOXC6 and PC9-HOXC6. Control cell lines transfected with the empty vector were named A549-NEO and PC9-NEO. Transfection efficiency was then assessed by qRT-PCR and Western blot (Fig. $2 \mathrm{~A}$ and $2 \mathrm{~B})$. We next examined the effect of HOXC6 on the growth of NSCLC cells, by using an image-based proliferation assay. As shown in Figure $2 \mathrm{C}$, the proliferation rate was significantly increased in HOXC6-overexpressing A549 and PC9 cells in comparison to control cells. We also utilized the CFSE assay as a secondary proliferation assay to validate this observation. There was an obvious shift in the fluorescence signal in A549-HOXC6 and PC9-HOXC6 cells 48 hours after CFSE labeling, indicating that $\mathrm{HOXC6}$ promoted the proliferation of both cell lines (Fig. 2D). These results demonstrate that overexpression of HOXC6 increased the proliferation of NSCLC cells.

\section{The promotion of migration and invasion by HOXC6 in NSCLC cells}

Migration and invasion are recognized as important hallmarks of cancer (Hanahan \& Weinberg 2011). To investigate the effect of HOXC6 on the migration and invasion of NSCLC cell lines, we made use of the wound healing and Matrigel assays. We observed significantly faster motility in A549-HOXC6 and PC9-HOXC6 cells in comparison to A549-NEO and PC9-NEO control cells in the wound healing experiment (Fig. 3A and 3B). Additionally, we observed that HOXC6 increased the invasive capacity of NSCLC cells in Matrigel as shown in Figure 3C and 3D. These results suggest that HOXC6 may have a pro-tumorigenic role in NSCLC.

\section{The downstream targets of HOXC6 revealed by gene expression profiling in NSCLC cells} Just like other members of the homeobox superfamily, HOXC6 is a transcription factor that regulates the expression of several downstream target genes. RNA-seq analysis was performed to identify genes modulated by HOXC6 in NSCLC cells. The results (GSE121896) showed that there were 310 genes upregulated and 665 genes downregulated in A549-HOXC6 cells in comparison to A549-NEO control cells. In addition, we found that there were 112 genes upregulated and 385 genes downregulated in PC9-HOXC6 cells in comparison to PC9-NEO control cells (Fig. 4A). Gene Ontology analysis indicated that these genes modulated by HOXC6 
226 may take part in various biological processes, such as morphogenesis and development, which is 227 consistent with the previously reported functions of the homeobox gene superfamily (Fig. 4B).

228 When we compared the genes regulated by HOXC6 in the 2 cell lines examined in this study, 229 there were 9 common upregulated genes and 133 common downregulated genes (Fig. 4C). 230 However, most of the genes whose expression levels were changed after HOXC6 transfection 231 were different in these two cell lines. This unexpected result suggests that transcriptional 232 regulation by HOXC6 is cell context-dependent.

\section{Pro-tumorigenic genes controlled by HOXC6 in NSCLC cells}

234 Gene Ontology analysis did not clearly identify the molecular mechanisms underlying the pro235 tumorigenic effect of HOXC6. Therefore, we supplemented this analysis by manually screening 236 critical tumor-associated HOXC6-upregulated genes using functional analysis and literature 237 review. Using this approach, we successfully identified several genes closely associated with 238 tumor progression. CEACAM6, SPARC, WNT6, CST1, and MMP2 were identified in A549239 HOXC6 cells, while CEACAM6 and KRT13 were identified in PC9-HOXC6 cells. These genes 240 have well documented roles in promoting cancer development (Table 1). We further analyzed the 241 expression of these genes in various cancers through GEPAI (Tang et al. 2017). The results 242 clearly demonstrate that the expression of these genes is associated with the progression of 243 cancers (Fig. 5A), and that they are primarily involved in the regulation of cell proliferation, 244 migration, and invasion. Furthermore, when we tried to analyze the function of the common 245 upregulated genes, i.e., HOXC6, SGK1, S100A4, MALL, CEACAM6, SLCO4A1-AS1, C11orf86, $246 E N S G 00000268621$, and ENSG00000129270, at least 5 of them, i.e., SGK1, S100A4, MALL, 247 CEACAM6 and SLCO4A1-AS1, have been reported to be implicated in the malignant phenotype 248 of various cancers, while the remaining genes have not been well studied (Egeland EV et al. 249 2017; Liang X et al. 2017; Rizeq et al. 2018; Yu J et al. 2018). We also analyzed the common 250 downregulated genes and found that many of these genes such as ANKRD12, KIAA1551, and $251 A P C$ encode proteins with tumor suppressive functions (Bai $\mathrm{R}$ et al. 2013; Cheng $\mathrm{J}$ et al. 2017; 252 Lv ZD et al. 2019). These data indicate that HOXC6 could exert its oncogenic function by both 253 activation of oncogenes and downregulation of the expression of tumor suppressive genes.

\section{Discussion}

255 Lung cancer is the most frequently occurring malignancy worldwide. Due to the lack of effective 256 measures for early diagnosis and treatment, the 5-year overall survival rate for NSCLC patients 257 is only $16 \%-18 \%$ (Siegel et al. 2018). The development of NSCLC involves both environmental 258 259 260 and genetic changes, and the activation of oncogenes is also an important factor. Therefore, the identification of novel biomarkers is critical for the improvement of clinical outcomes for NSCLC patients. 
261 In recent years, it has been found that the HOX gene is closely associated with the development

262 and prognosis of various cancers. HOXC6 is a member of the HOX family that acts as a

263 transcription factor and participates in the regulation of a number of genes during development.

264 HOXC6 has also been found to be highly expressed in several cancers. In this report, we provide

265 several lines of evidence demonstrating that HOXC6 plays an oncogenic role in human NSCLC.

266 First, HOXC6 expression was shown to be elevated in NSCLC tissues. Second, HOXC6

267 promoted the proliferation of NSCLC cells. Third, HOXC6 enhanced the migration and invasion

268 of NSCLC cells, which are fundamental hallmarks of cancer. Fourth, HOXC6 can upregulate the

269 expression of genes critical for the development and progression of various cancers. Therefore,

270 our work has established the basis for further investigation of HOXC6 and its oncogenic roles in

271 NSCLC.

272 To gain insight into the molecular mechanisms underlying the pro-tumorigenic functions of

273 HOXC6, RNA-seq was performed to identify its downstream targets. A number of genes have

274 been found to be modulated by HOXC6, but interestingly, we observed that there was very little

275 overlap in the genes modulated by HOXC6 in two NSCLC cell lines. This result suggests that

276 there is a cell context-dependent mechanism underlying the function of HOXC6. Since most

277 transcription factors need to form complexes to specifically regulate their target genes, the cell

278 context-specificity of HOXC6 may be caused by binding to different cofactors in different cell

279 lines. Despite the fact that different sets of genes are regulated by HOXC6 in various cell lines,

280 transfection of HOXC6 into both cell lines used in this study generated similar phenotypic

281 effects. Consistent with this result, we found that HOXC6 is a master regulator of many genes,

282 which have documented pro-tumorigenic functions. In our studies, we identified several genes,

283 including CEACAM6, SPARC, WNT6, CST1, MMP2, and KRT13, which have been extensively

284 studied and demonstrated to be involved in the regulation of tumor growth, migration and

285 invasion, cell cycle, and apoptosis (Chang et al. 2018; Choi et al. 2009; Dai et al. 2017; Kuo et

286 al. 2014; Rizeq et al. 2018; Wang et al. 2018; Yusuf et al. 2014; Zheng \& Yu 2018). In other

287 types of cancers, HOXC6 has also been shown to have the capacity to regulate these cancer-

288 related genes. For example, HOXC6 directly regulates gene expression of Bone morphogenetic

289 protein 7 (BMP7), Fibroblast growth factor receptor 2 (FGF2), and Platelet-derived growth

290 factor receptor (PDGFR) in prostate cancer (McCabe et al. 2008). HOXC6 promotes the

291 migration, invasion, and progression of gastric cancer by upregulating Matrix metalloproteinase

2929 (MMP9) (Chen et al. 2016a). These results suggest that HOXC6 can promote tumorigenesis by

293 regulating distinct sets of genes in various cellular contexts.

\section{Conclusion}


295 In conclusion, we demonstrate that HOXC6 was highly expressed in NSCLC tissues and 296 correlated with the malignant phenotype of NSCLC cells. In addition, bioinformatics analyses 297 showed that HOXC6 may enhance lung cancer progression by regulating the expression of pro298 tumorigenic genes involved in proliferation, migration, and invasion. Our study highlighted the 299 oncogenic potential of HOXC6 and suggests that it is a candidate molecular marker for the 300 diagnosis and treatment of NSCLC.

301

302 303

304 305 306 307 308 309 310 311 312 313 314 315 316 317 318 319 320 321 322 323 324 325 326 327

\section{References}

Anders S, Pyl PT, and Huber W. 2015. HTSeq--a Python framework to work with highthroughput sequencing data. Bioinformatics 31:166-169. 10.1093/bioinformatics/btu638

Apiou F, Flagiello D, Cillo C, Malfoy B, Poupon MF, and Dutrillaux B. 1996. Fine mapping of human HOX gene clusters. Cytogenet Cell Genet 73:114-115. 10.1159/000134320

Bai R, Li D, Shi Z, Fang X, Ge W, Zheng S. 2013. Clinical significance of Ankyrin repeat domain 12 expression in colorectal cancer. J Exp Clin Cancer Re 32(1):35.

Care A, Felicetti F, Meccia E, Bottero L, Parenza M, Stoppacciaro A, Peschle C, and Colombo MP. 2001. HOXB7: a key factor for tumor-associated angiogenic switch. Cancer Res 61:6532-6539.

Chang CH, Yen MC, Liao SH, Hsu YL, Lai CS, Chang KP, and Hsu YL. 2018. Correction: Chang, C.-H.; et al. Secreted Protein Acidic and Rich in Cysteine (SPARC) Enhances Cell Proliferation, Migration, and Epithelial Mesenchymal Transition, and SPARC Expression Is Associated with Tumor Grade in Head and Neck Cancer. Int. J. Mol. Sci. 2017, 18, 1556. Int J Mol Sci 19. 10.3390/ijms19082338

Chen SW, Zhang Q, Xu ZF, Wang HP, Shi Y, Xu F, Zhang WJ, Wang P, and Li Y. 2016 a. HOXC6 promotes gastric cancer cell invasion by upregulating the expression of MMP9. Mol Med Rep 14:3261-3268. 10.3892/mmr.2016.5640

Chen W, Zheng R, Baade PD, Zhang S, Zeng H, Bray F, Jemal A, Yu XQ, and He J. 2016 b. Cancer statistics in China, 2015. CA Cancer J Clin 66:115-132.

Cheng J, Demeulemeester J, Wedge DC, Vollan HKM, Pitt JJ, Russnes HG, Pandey BP, Nilsen G, Nord S, Bignell GR, White KP, Børresen-Dale AL, Campbell PJ, Kristensen VN, Stratton MR, Lingjærde OC, Moreau Y, Van Loo P. 2017. Pan-cancer analysis of homozygous deletions in primary tumours uncovers rare tumour suppressors. Nat Commun 8(1):1221

Choi EH, Kim JT, Kim JH, Kim SY, Song EY, Kim JW, Kim SY, Yeom YI, Kim IH, and Lee HG. 2009. Upregulation of the cysteine protease inhibitor, cystatin SN, contributes to cell 
328

329

330

331

332

333

334

335

336

337

338

339

340

341

342

343

344

345

346

347

348

349

350

351

352

353

354

355

356

357

358

359

360

361

362

363

proliferation and cathepsin inhibition in gastric cancer. Clin Chim Acta 406:45-51. 10.1016/j.cca.2009.05.008

Chu MC, Selam FB, and Taylor HS. 2004. HOXA10 regulates p53 expression and matrigel invasion in human breast cancer cells. Cancer Biol Ther 3:568-572.

Cillo C, Cantile M, Faiella A, and Boncinelli E. 2001. Homeobox genes in normal and malignant cells. J Cell Physiol 188:161-169. 10.1002/jcp.1115

Dai DN, Li Y, Chen B, Du Y, Li SB, Lu SX, Zhao ZP, Zhou AJ, Xue N, Xia TL, Zeng MS, Zhong Q, and Wei WD. 2017. Elevated expression of CST1 promotes breast cancer progression and predicts a poor prognosis. J Mol Med (Berl) 95:873-886. 10.1007/s00109-017-1537-1

DeInnocentes P, Perry AL, Graff EC, Lutful Kabir FM, and Curtis Bird R. 2015. Characterization of HOX gene expression in canine mammary tumour cell lines from spontaneous tumours. Vet Comp Oncol 13:322-336. 10.1111/vco.12062

Egeland EV, Boye K, Park D, Synnestvedt M, Sauer T; Oslo Breast Cancer Consortium (OSBREAC), Naume B, Borgen E, Mælandsmo GM. 2017. Prognostic significance of S100A4-expression and subcellular localization in early-stage breast cancer. Breast Cancer Res Treat 162(1):127-137.

Feng JQ, Liu WJ, Chen HY, Guo QL, Chen A, and Chen SQ. 2009. [Effect of human cytomegalovirus infection on the expression of hoxc4 and hoxc6 genes in the proliferation of lymphocytic progenitor cells]. Zhongguo Shi Yan Xue Ye Xue Za Zhi 17:141-145.

Hanahan D, and Weinberg RA. 2011. Hallmarks of cancer: the next generation. Cell 144:646674. 10.1016/j.cell.2011.02.013

Harrow J, Frankish A, Gonzalez JM, Tapanari E, Diekhans M, Kokocinski F, Aken BL, Barrell D, Zadissa A, Searle S, Barnes I, Bignell A, Boychenko V, Hunt T, Kay M, Mukherjee G, Rajan J, Despacio-Reyes G, Saunders G, Steward C, Harte R, Lin M, Howald C, Tanzer A, Derrien T, Chrast J, Walters N, Balasubramanian S, Pei B, Tress M, Rodriguez JM, Ezkurdia I, van Baren J, Brent M, Haussler D, Kellis M, Valencia A, Reymond A, Gerstein M, Guigo R, and Hubbard TJ. 2012. GENCODE: the reference human genome annotation for The ENCODE Project. Genome Res 22:1760-1774.

$10.1101 /$ gr. 135350.111

Ji M, Feng Q, He G, Yang L, Tang W, Lao X, Zhu D, Lin Q, Xu P, Wei Y, and Xu J. 2016. Silencing homeobox C6 inhibits colorectal cancer cell proliferation. Oncotarget 7:2921629227. 10.18632/oncotarget.8703

Kim D, Langmead B, and Salzberg SL. 2015. HISAT: a fast spliced aligner with low memory requirements. Nat Methods 12:357-360. 10.1038/nmeth.3317 
364

365

366

367

368

369

370

371

372

373

374

375

376

377

378

379

380

381

382

383

384

385

386

387

388

389

390

391

392

393

394

395

396

397

398

Kuo HY, Huang YS, Tseng CH, Chen YC, Chang YW, Shih HM, and Wu CW. 2014. PML represses lung cancer metastasis by suppressing the nuclear EGFR-mediated transcriptional activation of MMP2. Cell Cycle 13:3132-3142. $10.4161 / 15384101.2014 .949212$

Lee SH, Suh IB, Lee EJ, Hur GY, Lee SY, Lee SY, Shin C, Shim JJ, In KH, Kang KH, Yoo SH, and Kim JH. 2013. Relationships of coagulation factor XIII activity with cell-type and stage of non-small cell lung cancer. Yonsei Med J 54:1394-1399. 10.3349/ymj.2013.54.6.1394

Liang X, Lan C, Jiao G, Fu W, Long X, An Y, Wang K, Zhou J, Chen T, Li Y, Xu J, Huang Q, $\mathrm{Xu}$ B, Xiao J. 2017. Therapeutic inhibition of SGK1 suppresses colorectal cancer. Exp Mol Med 49(11):e399.

Love MI, Huber W, and Anders S. 2014. Moderated estimation of fold change and dispersion for RNA-seq data with DESeq2. Genome Biol 15:550. 10.1186/s13059-014-0550-8

Lv ZD, Xin HN, Yang ZC, Wang WJ, Dong JJ, Jin LY, Li FN. 2019. miR-135b promotes proliferation and metastasis by targeting APC in triple-negative breast cancer. $J$ Cell Physiol.

Maroulakou IG, and Spyropoulos DD. 2003. The study of HOX gene function in hematopoietic, breast and lung carcinogenesis. Anticancer Res 23:2101-2110.

McCabe CD, Spyropoulos DD, Martin D, and Moreno CS. 2008. Genome-wide analysis of the homeobox C6 transcriptional network in prostate cancer. Cancer Res 68:1988-1996. 10.1158/0008-5472.CAN-07-5843

Moon SM, Kim SA, Yoon JH, and Ahn SG. 2012. HOXC6 is deregulated in human head and neck squamous cell carcinoma and modulates Bcl-2 expression. J Biol Chem 287:3567835688. 10.1074/jbc.M112.361675

National Lung Screening Trial Research T, Aberle DR, Adams AM, Berg CD, Black WC, Clapp JD, Fagerstrom RM, Gareen IF, Gatsonis C, Marcus PM, and Sicks JD. 2011. Reduced lung-cancer mortality with low-dose computed tomographic screening. $N$ Engl J Med 365:395-409. 10.1056/NEJMoa1102873

Rizeq B, Zakaria Z, and Ouhtit A. 2018. Towards understanding the mechanisms of actions of carcinoembryonic antigen-related cell adhesion molecule 6 in cancer progression. Cancer Sci 109:33-42. 10.1111/cas.13437

Shah N, and Sukumar S. 2010. The Hox genes and their roles in oncogenesis. Nat Rev Cancer 10:361-371. 10.1038/nrc2826

Siegel RL, Miller KD, and Jemal A. 2018. Cancer statistics, 2018. CA Cancer J Clin 68:7-30. $10.3322 /$ caac. 21442 
399

400

401

402

403

404

405

406

407

408

409

410

411

412

413

414

415

416

417

418

419

420

421

422

423

424

425

426

427

428

429

430

431

432

433

Sui CJ, Xu F, Shen WF, Dai BH, Lu JJ, Zhang MF, and Yang JM. 2016. MicroRNA-147 suppresses human hepatocellular carcinoma proliferation migration and chemosensitivity by inhibiting HOXC6. Am J Cancer Res 6:2787-2798.

Tang Z, Li C, Kang B, Gao G, Li C, Zhang Z. GEPIA: a web server for cancer and normal gene expression profiling and interactive analyses, 2017. Nucleic Acids Res 45(W1):W98W102. 10.1093/nar/gkx247

Vinarskaja A, Yamanaka M, Ingenwerth M, and Schulz WA. 2011. DNA Methylation and the HOXC6 Paradox in Prostate Cancer. Cancers (Basel) 3:3714-3725. 10.3390/cancers3043714

Wang X, Yang B, She Y, and Ye Y. 2018. The lncRNA TP73-AS1 promotes ovarian cancer cell proliferation and metastasis via modulation of MMP2 and MMP9. J Cell Biochem 119:7790-7799. 10.1002/jcb.27158

Wright CV, Cho KW, Hardwicke J, Collins RH, and De Robertis EM. 1989. Interference with function of a homeobox gene in Xenopus embryos produces malformations of the anterior spinal cord. Cell 59:81-93.

Ye B, and Zhao H. 2016. [Revision of the TNM Stage Grouping in the Forthcoming Eighth Edition of the TNM Classification for Lung Cancer]. Zhongguo Fei Ai Za Zhi 19:337342. 10.3779/j.issn.1009-3419.2016.06.07

Yu G, Wang LG, Han Y, and He QY. 2012. clusterProfiler: an R package for comparing biological themes among gene clusters. OMICS 16:284-287. 10.1089/omi.2011.0118

Yu J, Han Z, Sun Z, Wang Y, Zheng M, Song C. 2018. LncRNA SLCO4A1-AS1 facilitates growth and metastasis of colorectal cancer through $\beta$-catenin-dependent Wnt pathway. $J$ Exp Clin Cancer Res 37(1):222.

Yusuf N, Inagaki T, Kusunoki S, Okabe H, Yamada I, Matsumoto A, Terao Y, Takeda S, and Kato K. 2014. SPARC was overexpressed in human endometrial cancer stem-like cells and promoted migration activity. Gynecol Oncol 134:356-363. 10.1016/j.ygyno.2014.04.009

Zhang Q, Jin XS, Yang ZY, Wei M, Liu BY, and Gu QL. 2013. Upregulated Hoxc6 expression is associated with poor survival in gastric cancer patients. Neoplasma 60:439-445. 10.4149/neo_2013_057

Zheng XL, and Yu HG. 2018. Wnt6 contributes tumorigenesis and development of colon cancer via its effects on cell proliferation, apoptosis, cell-cycle and migration. Oncol Lett 16:1163-1172. 10.3892/ol.2018.8729

Zienolddiny S, and Skaug V. 2011. Single nucleotide polymorphisms as susceptibility, prognostic, and therapeutic markers of nonsmall cell lung cancer. Lung Cancer 3:1. 


\section{Figure 1}

Elevated expression of HOXC6 in NSCLC in comparison to adjacent normal tissues.

(A, B) The bioinformatic analysis of publicly available gene expression data sets for HOXC6 expression in lung cancer tissues. Microarray dataset GSE30219 (normal=15, cancer=39) and GSE27262 (normal=25, cancer=25) were download from Gene Expression Omnibus(GEO). HOXC6 expression distribution in Fig. 1A and 1B, with a fold change of 5 (q.value $=1.4332 \mathrm{e}-07$ ) and 8.8 (q.value $=0.0039$ ), which were calculated by $\mathrm{R}$ package limma (Ritchie et al. 2015). (C) Relative expression of HOXC6 mRNA in 30 NSCLC and paired adjacent normal tissues as determined by qRT-PCR. The data were assessed using Chi-square test. (D) Expression of HOXC6 protein as determined by IHC. The IHC score of HOXC6 was calculated as the staining intensity $(0,1,2$, or 3$) \times$ the staining extent $(0-100 \%)$. We compared matched samples as in Fig. 1D using paired two-tailed Student's $t$ test. (E) Representative images of IHC staining of HOXC6 in NSCLC tumor (T) and adjacent normal tissues $(\mathrm{N})$, Scale bar $=200 \mu \mathrm{m}$. 
A

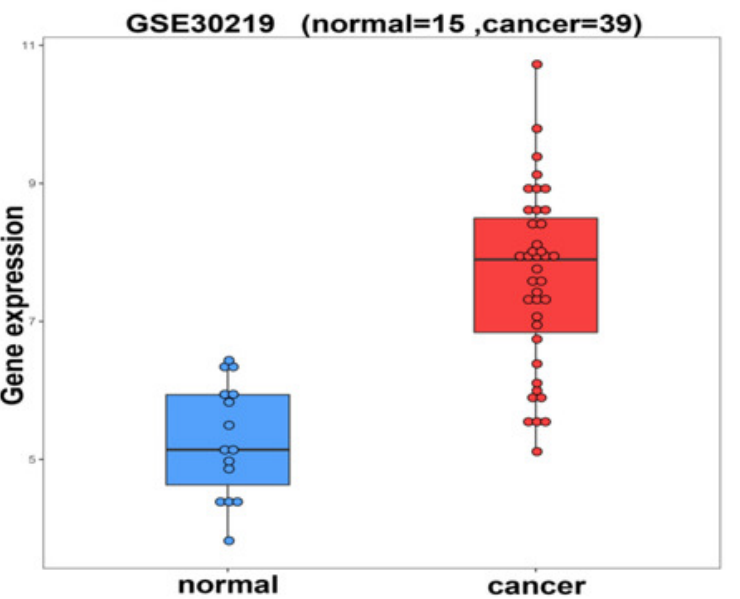

C

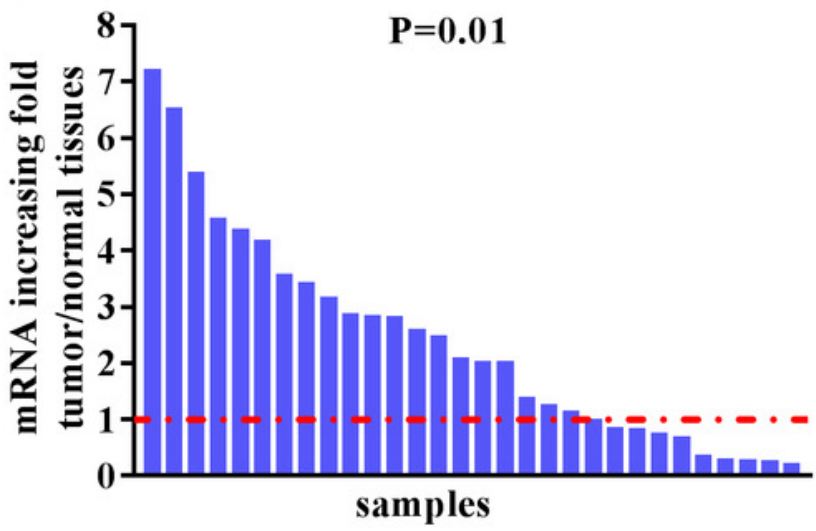

E

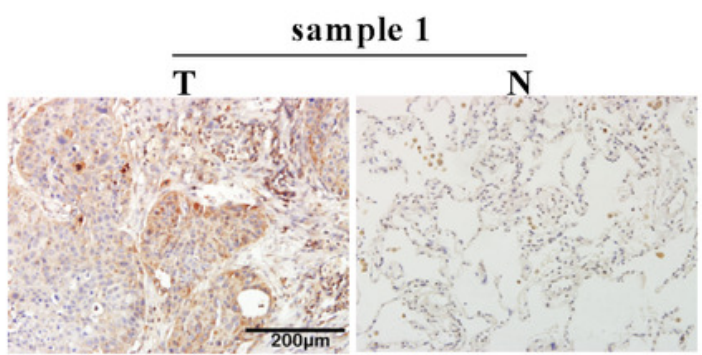

G

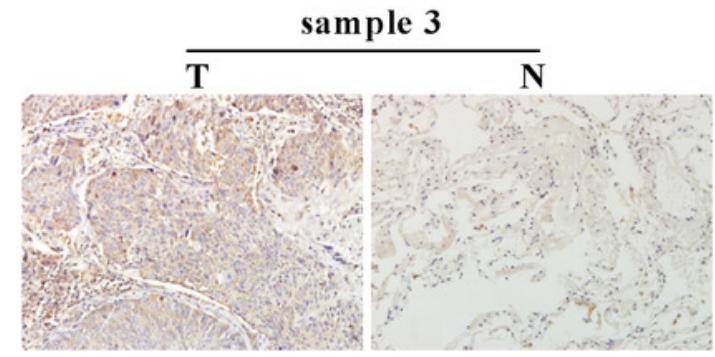

B

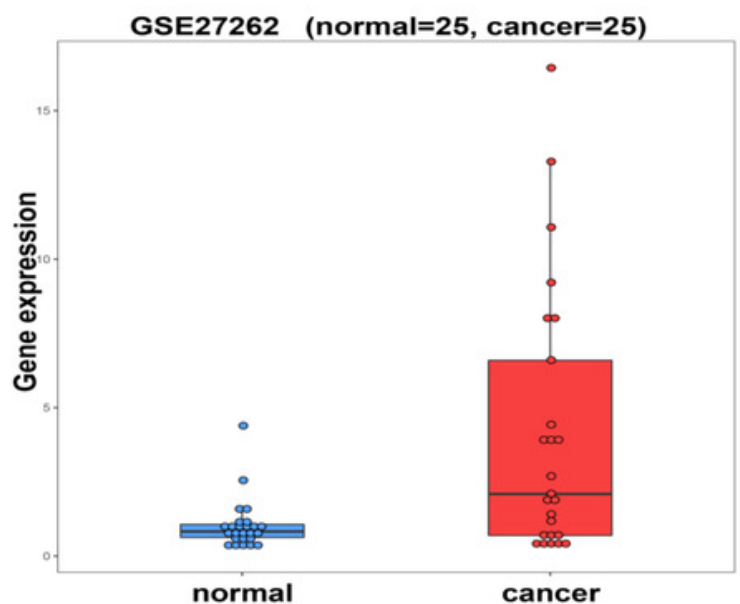

D

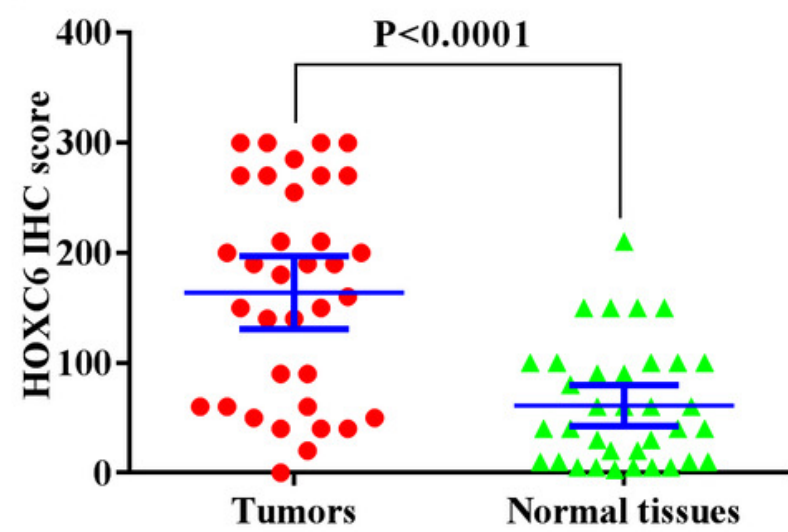

F

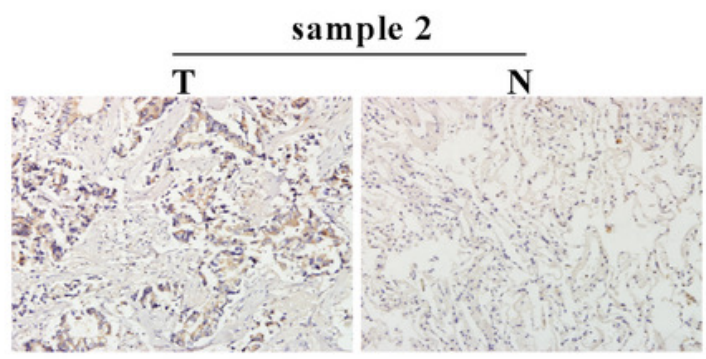

H

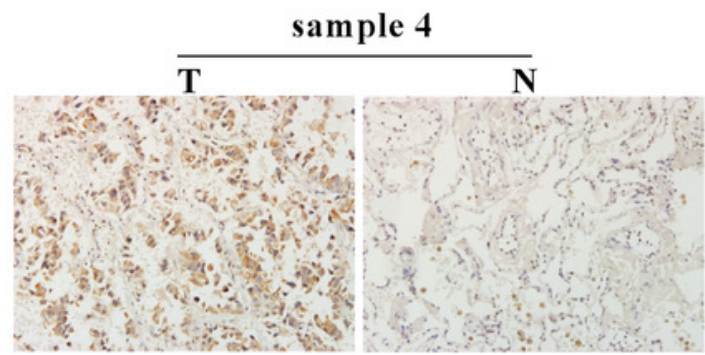




\section{Figure 2}

Overexpression of HOXC6 promotes NSCLC cell proliferation.

(A, B) Western Blot analysis of HOXC6 in A549 and PC9 HOXC6-expressing or control cells.

(C) IncuCyte Live Cell Imaging System analysis of NSCLC cell lines A549 and PC9 transfected with HOXC6 or NEO lentiviral vectors. Cells $\left(1 \times 10^{4}\right)$ were seeded in 96 -well plates and monitored at 2-hour intervals, 3 replicates for each sample. (D) Flow cytometry analysis of CFSE-labeled HOXC6-expressing or control NSCLC cell lines, 3 replicates for each sample. 


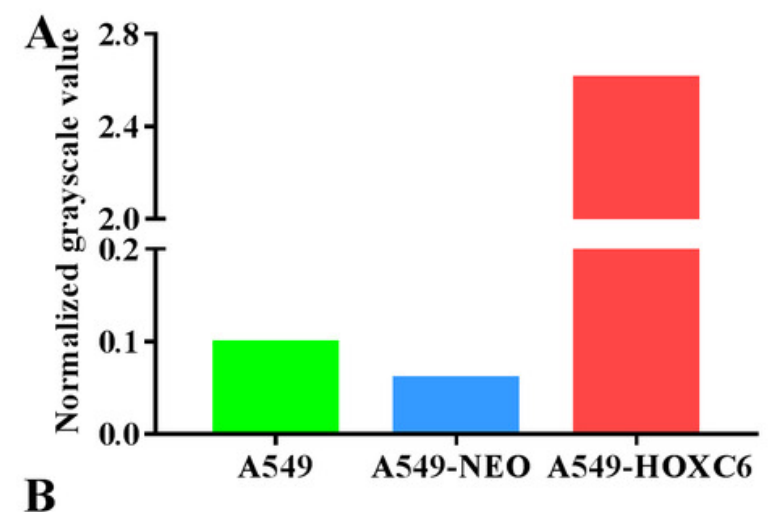

HoXc6

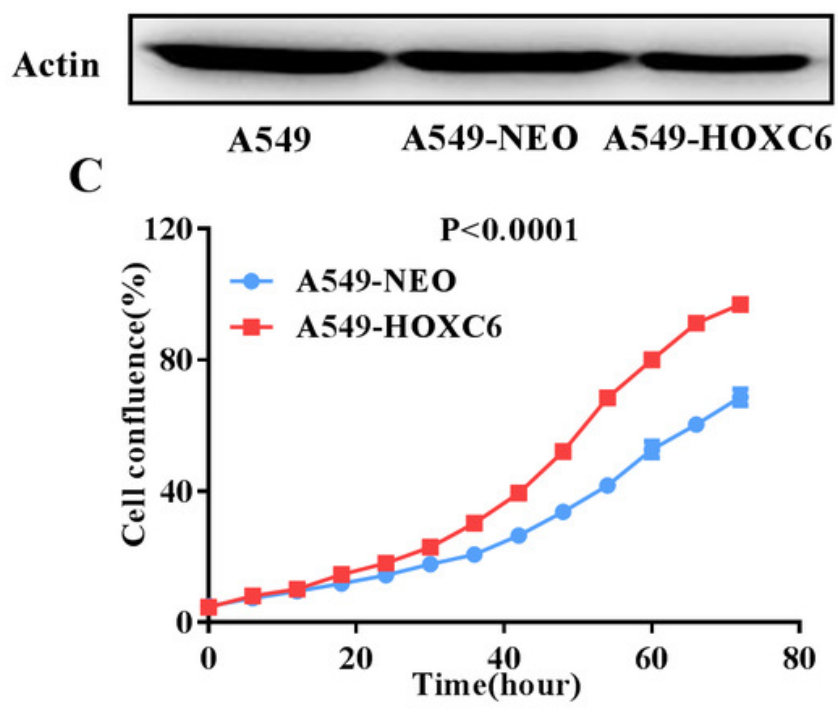

D

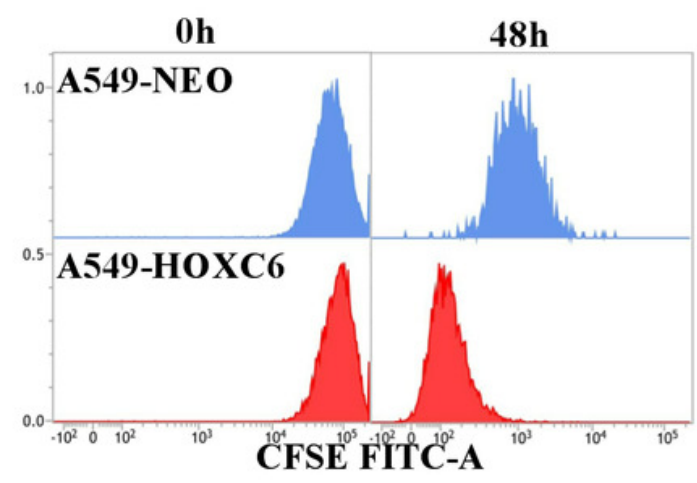

$\mathbf{E}$

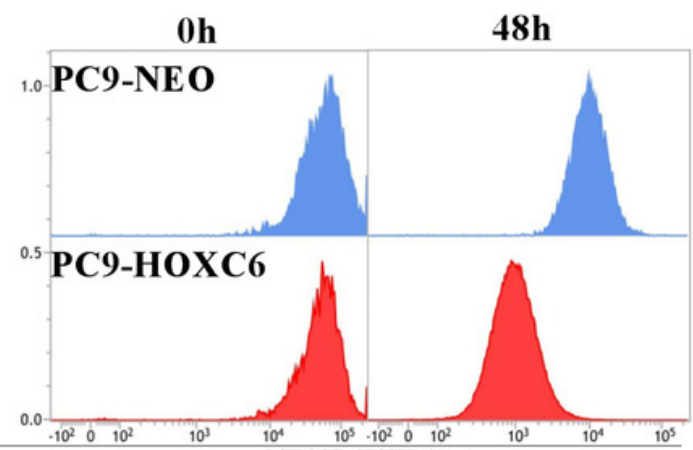

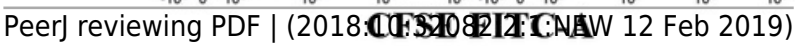
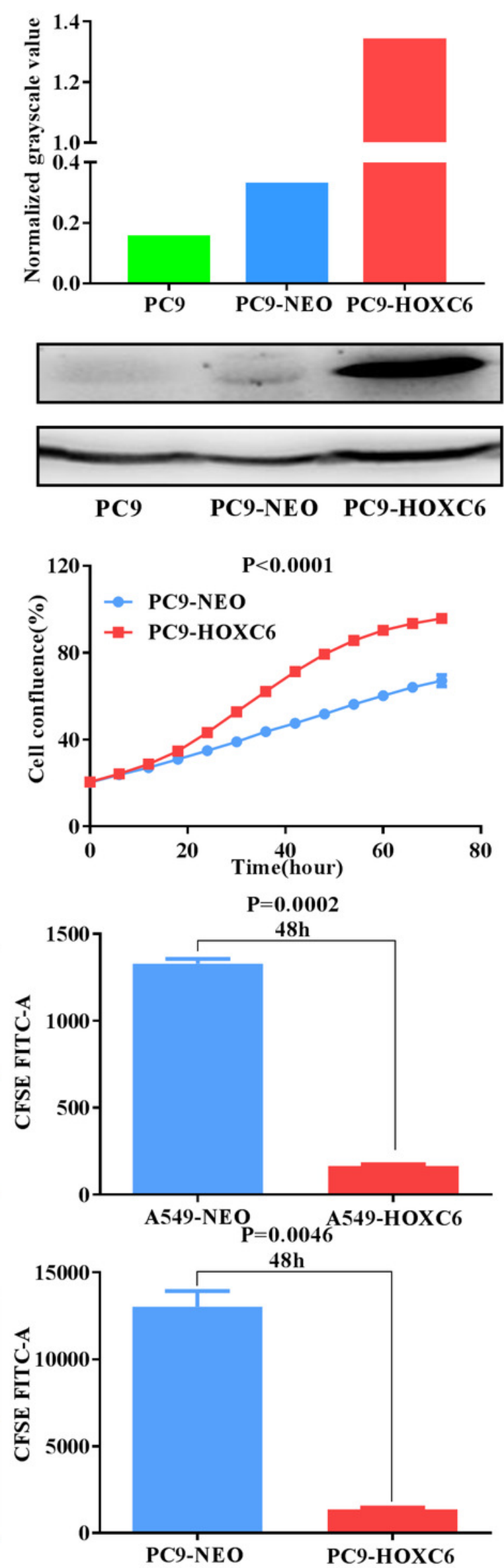


\section{Figure 3}

Overexpression of HOXC6 promotes migration and invasion of NSCLC cells.

(A, B) Wound healing assay of A549 and PC9 cells expressing HOXC6 or NEO. Scale bar $=150 \mu \mathrm{m}$. (C, D) Invasion of HOXC6-expressing or control A549 and PC9 cells was assessed in a wound healing assay using 30\% Matrigel. Scale bar $=150 \mu \mathrm{m}$. 

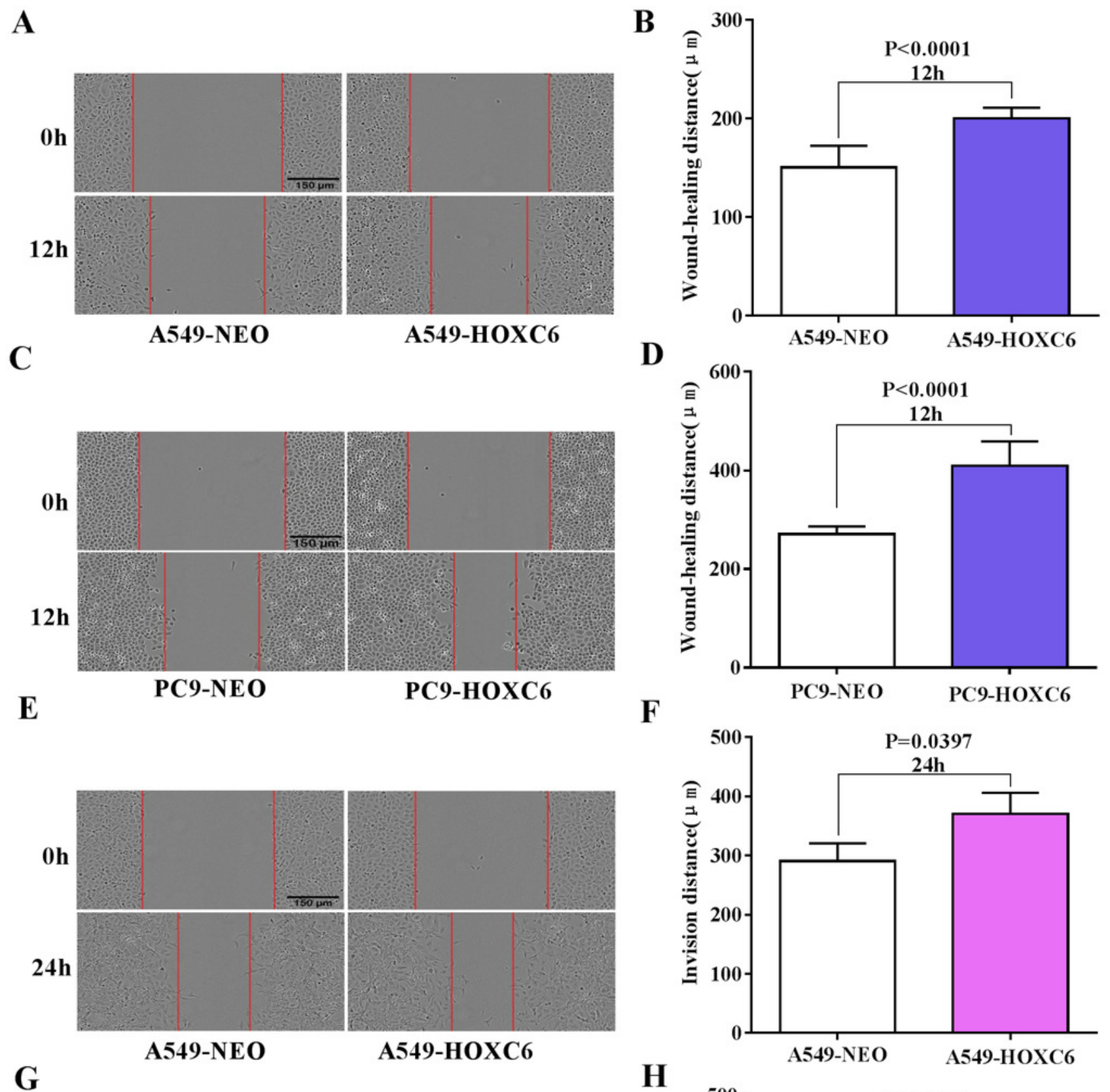

F
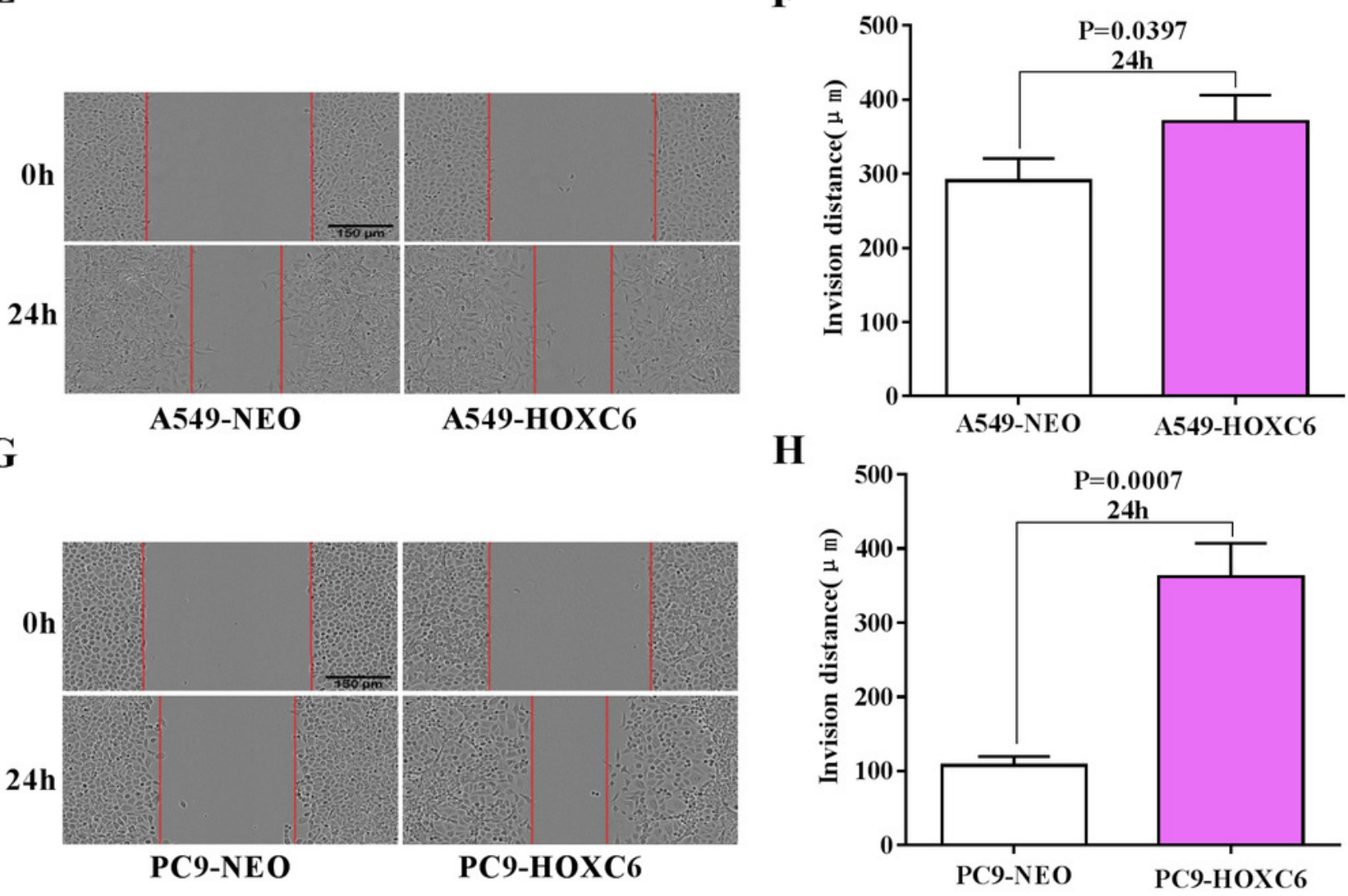


\section{Figure 4}

Analysis of RNA-seq.

(A) The volcano map shows 310 upregulated genes and 665 downregulated genes in A549HOXC6 cells in comparison to A549-NEO cells, and 112 upregulated genes and 385 downregulated genes in PC9-HOXC6 cells in comparison to PC9-NEO cells. Log2 fold change (Log2FC) $>1$ or $<-1$, and $P<0.05$ were considered to be statistically significant. (B) Gene ontology (GO) analysis identified biological processes impacted by HOXC6-regulated genes in NSCLC cells. (C) Overlap of upregulated and downregulated genes in A549-HOXC6 and PC9HOXC6 cells. 
A

C
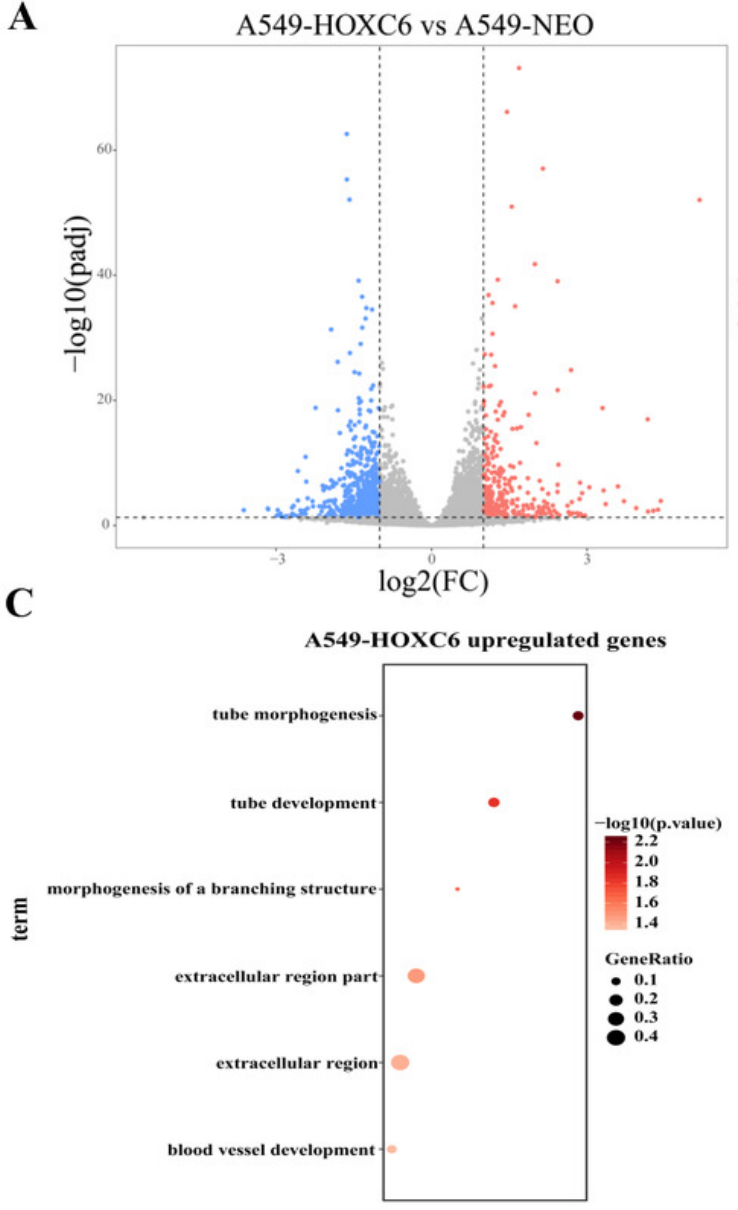

$\mathbf{E}$

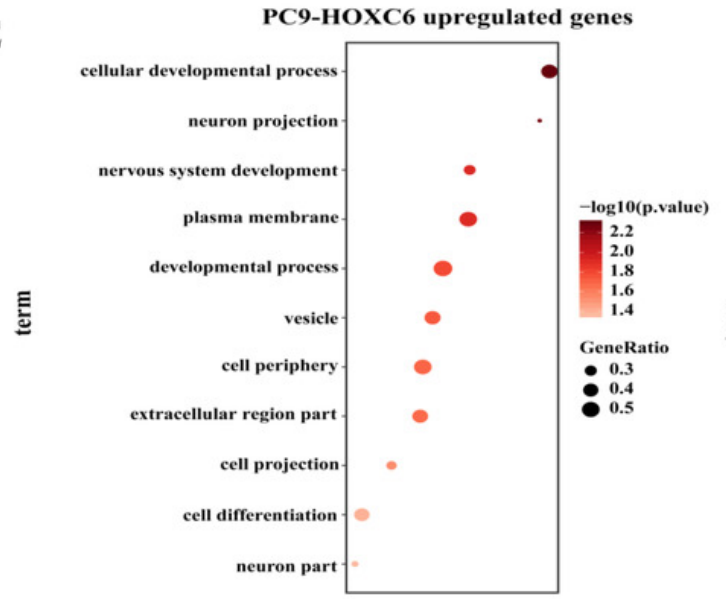

G

Up-regulated genes

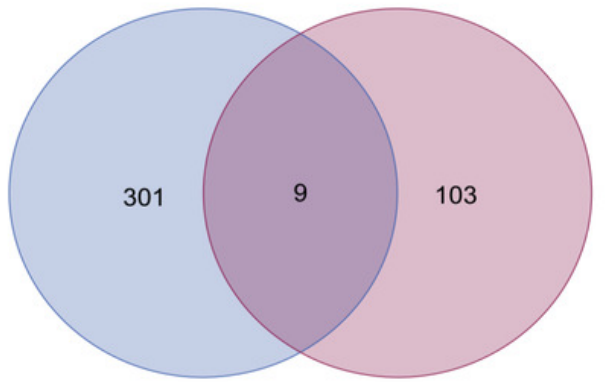

A549-HOXC6 vs PC9-HOXC6

PeerJ reviewing PDF | (2018:10:32082:2:1:NEW 12 Feb 2019)
B $\quad$ PC9-HOXC6 vs PC9-NEO
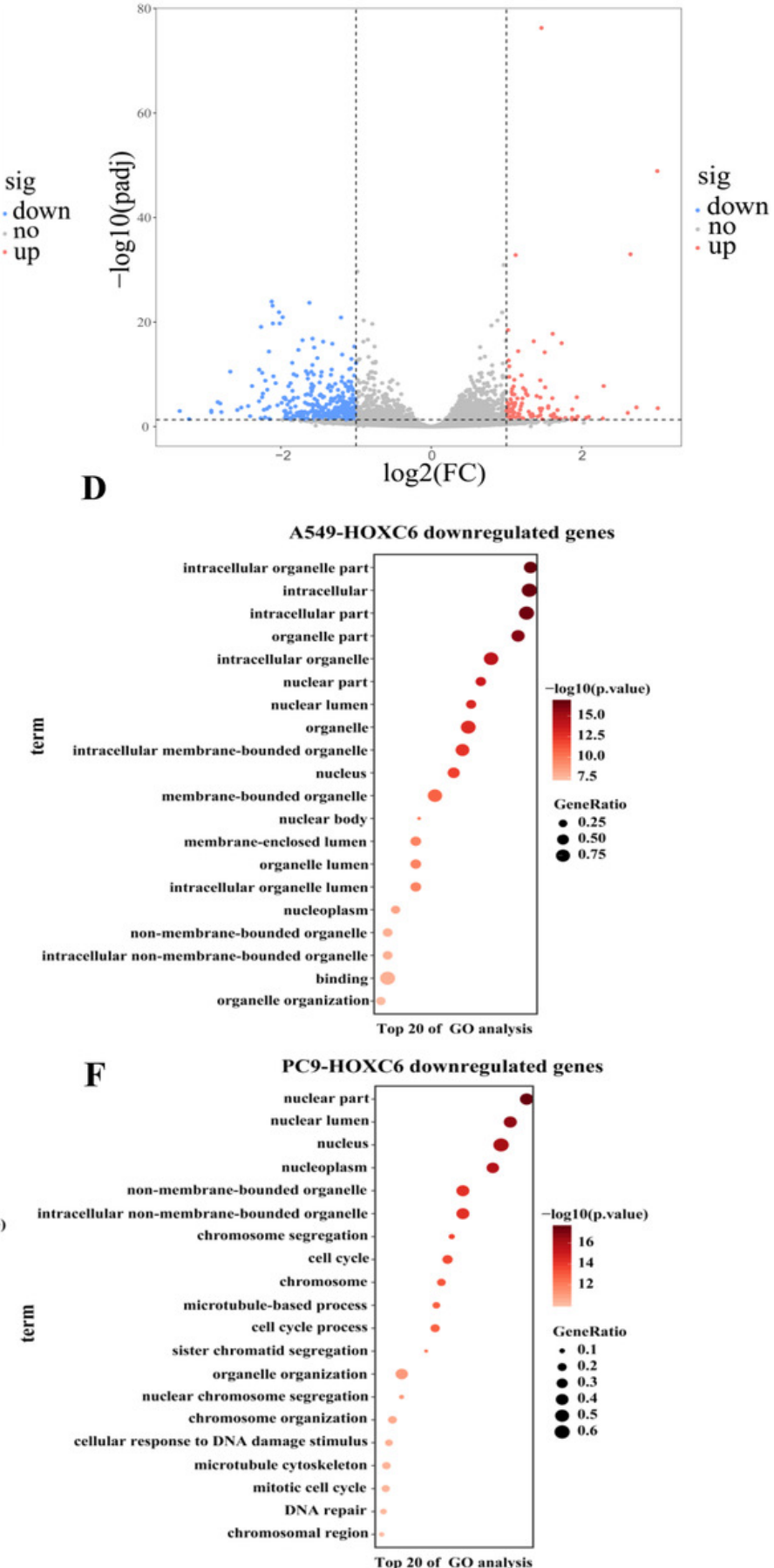

H Down-regulated genes

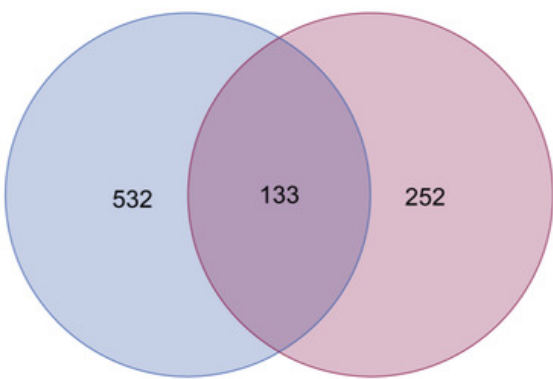

A549-HOXC6 vs PC9-HOXC6 


\section{Figure 5}

The expression of pro-tumorigenic genes in various cancers.

(A) The expression of CEACAM6, SPARC, WNT6, CST1, MMP2, and KRT13 in various cancers, $* P<0.05$. Breast invasive carcinoma (BRCA), Pancreatic adenocarcinoma (PAAD), Adrenocortical carcinoma (ACC), Cholangiocarcinoma (CHOL), Stomach adenocarcinoma (STAD), Lymphoid Neoplasm Diffuse Large B-cell Lymphoma (DLBC), Pheochromocytoma and Paraganglioma (PCPG), Colon adenocarcinoma (COAD), Lung squamous cell carcinoma (LUSC), Cervical squamous cell carcinoma and endocervical adenocarcinoma (CESC). 
A

C

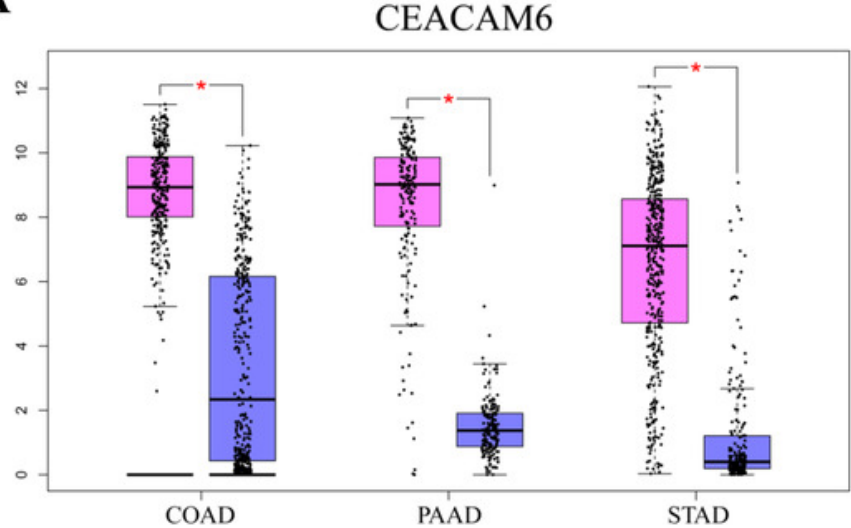

C

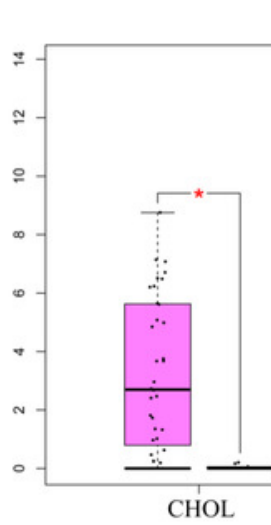

CST1

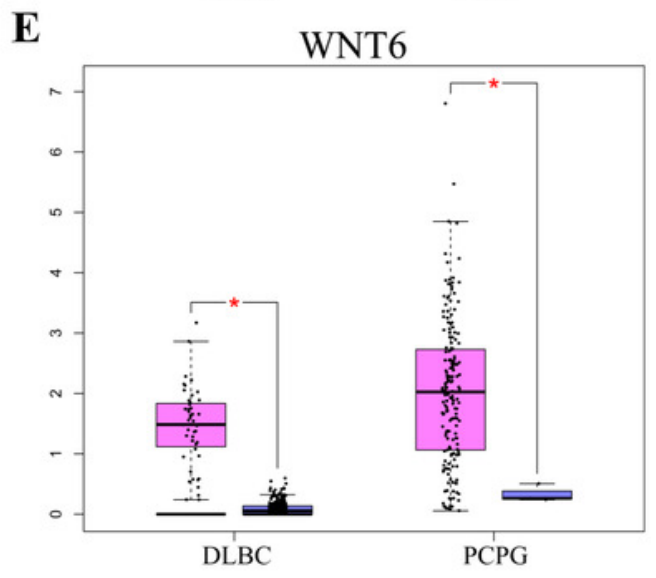

B

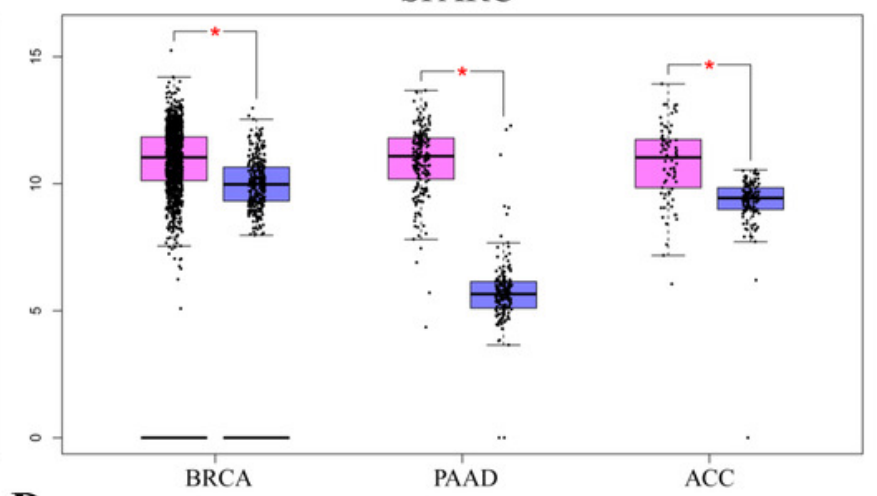

D

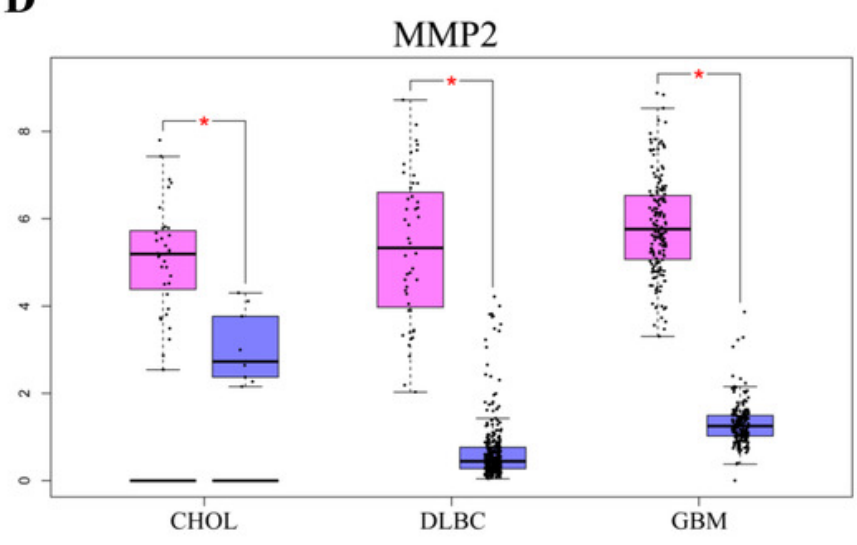
F $\quad$ KRT13

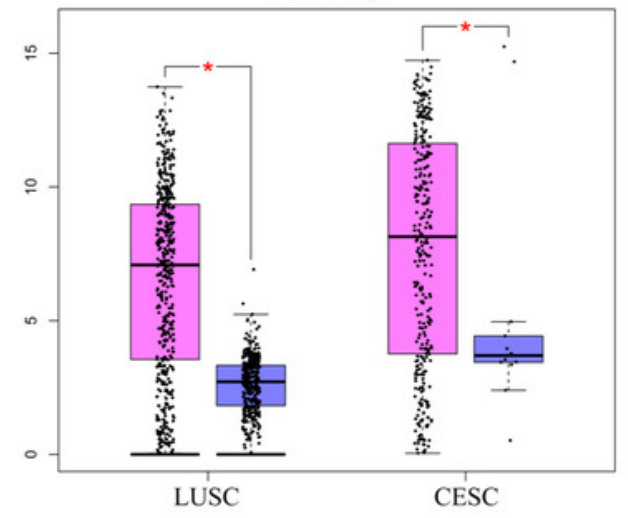




\section{Table $\mathbf{1}$ (on next page)}

Potential target genes of HOXC6 in NSCLC.

*Proliferation (P), Migration (M), Invasion (I), Angiogenesis (A), Cell cycle (CC), Tolerance (T), Stroma formation (SF). 
1

\begin{tabular}{|c|c|c|c|}
\hline Gene Symbol & Gene description & Gene function* & References \\
\hline CEACAM6 & Tumor marker & $\mathrm{A}, \mathrm{M}, \mathrm{I}, \mathrm{P}, \mathrm{T}$ & $\begin{array}{l}\text { Rizeq B et al. (2018) } \\
\text { Zang M et al. (2015) }\end{array}$ \\
\hline$S P A R C$ & Matrix-associated protein & $\mathrm{P}, \mathrm{M}, \mathrm{CC}, \mathrm{SF}$ & $\begin{array}{l}\text { Chang CH et al. (2018) } \\
\text { Yusuf N et al. (2014) }\end{array}$ \\
\hline WNT6 & $\begin{array}{l}\text { A family of highly conserved } \\
\text { developmental control genes }\end{array}$ & $\mathrm{P}, \mathrm{M}, \mathrm{A}, \mathrm{CC}, \mathrm{T}$ & $\begin{array}{l}\text { Yuan G et al. (2013) } \\
\text { Zheng XL et al.(2018) }\end{array}$ \\
\hline CST1 & The cystatin superfamily & $\mathrm{P}, \mathrm{M}, \mathrm{I}$ & $\begin{array}{l}\text { Choi EH et al. (2009) } \\
\text { Dai DN et al. (2017) }\end{array}$ \\
\hline$M M P 2$ & $\begin{array}{l}\text { The major structural component of } \\
\text { basement membranes }\end{array}$ & $\mathrm{M}, \mathrm{I}$ & $\begin{array}{l}\text { Kalhori V et al. (2015) } \\
\text { Kuo HY et al. (2014) }\end{array}$ \\
\hline KRT13 & $\begin{array}{l}\text { Encoded a member of the keratin } \\
\text { gene family }\end{array}$ & $\mathrm{M}, \mathrm{I}, \mathrm{CC}, \mathrm{A}$ & $\begin{array}{l}\text { LIU Man et al. (2014) } \\
\text { Li Q et al. (2016) }\end{array}$ \\
\hline
\end{tabular}

